\title{
Retrospective cohort follow-up study of individuals detained under Section 136
}

\author{
Jennifer L. Burgess, Sarah-Jane White and Aileen O'Brien
}

\section{Background}

An original cohort study found that over half of the individuals detained under Section 136 (S136) of the Mental Health Act 1983 were discharged home after assessment, and nearly half were intoxicated.

\section{Aims}

To investigate whether the cohort was followed up by psychiatric services, characterise those repeatedly detained and assess whether substance use was related to these outcomes.

\section{Method}

Data were retrospectively collected from the notes of 242 individuals, who presented after S136 detention to a place of safety over a 6-month period, and were followed up for 1 year

\section{Results}

After 1 year, $48 \%$ were in secondary care. Those with psychosis were the most likely to be admitted. Diagnoses of personality disorder or substance use were associated with multiple detentions; however, few were in contact with secondary services.

\section{Conclusions}

Crisis and long-term care pathways for these groups need to be developed to reduce repeated and unnecessary police detention.

\section{Declaration of interest}

None.

\section{Copyright and usage}

(c) The Royal College of Psychiatrists 2017. This is an open access article distributed under the terms of the creative Commons Non-Commercial, No Derivatives (CC BY-NC-ND) license.
Section 136 (S136) of the Mental Health Act 1983 (amended 2007) allows police officers to remove an individual whom they believe to be mentally disordered and in 'immediate need of care or control' from a public place to a designated place of safety, 'in the interest of that person or for the protection of other persons'. A place of safety is usually a unit within a psychiatric hospital ('Section 136 suite'), a police station or an Accident and Emergency department, and the individual can be detained for up to $72 \mathrm{~h}$ for the purpose of conducting a Mental Health Act assessment. The use of S136 has increased fourfold over the past 10 years, and 18\% between 2014/2015 and 2015/2016. ${ }^{1}$ Zisman \& O'Brien sought to characterise individuals detained under S136 by conducting a retrospective cohort study over a 6-month period at a London mental health trust. ${ }^{2}$ Two-hundred and forty-five people were detained and brought to one S136 suite between February 2012 and July 2012. More than half of the individuals had been previously detained under S136 or admitted to hospital. The most common reason for detention was threat to self-harm, but after a Mental Health Act assessment over half were discharged home. The study also found that $44.9 \%$ of individuals were intoxicated with alcohol or drugs. Being intoxicated was associated with a breach of the 6-hour assessment completion target and greater likelihood of being discharged home after assessment. This study aims to follow-up the original cohort for 1 year after their detention. It will provide more detailed information about the outcomes of the original assessments, whether the cohort went on to use local mental health services, and explore the characteristics of the group of patients who present to services repeatedly. In addition, it will investigate whether drug and alcohol intoxication was related to these outcomes.

\section{Method}

Data were retrospectively collected from the electronic notes of the original cohort. These 245 individuals presented to the South West
London and St George's Mental Health NHS Trust place of safety between 1 February and 31 July 2012, after being detained under S136 of the Mental Health Act. If the individual was detained multiple times during the data collection period, only the first detention was included in the analysis. The follow-up period was defined as 365 days from the index detention date as recorded in the notes. Three individuals were removed from the follow-up analysis as they were found to be duplicates.

Additional data were collected about the original cohort, including ICD-10 psychiatric diagnosis and outcome of the index S136 detention. ${ }^{3}$ The ICD-10 diagnosis code recorded at the time of the index detention was obtained from the individual's electronic notes, and whether or not they had a comorbid or secondary diagnosis of psychoactive substance use. Follow-up data collected included whether an individual had repeat detentions under S136, whether an individual was admitted to hospital and details of the admission (including admission resulting from the initial S136 detention), how many referrals were made to secondary psychiatric services and had at least one contact (excluding the event of hospital admission, and including referrals to liaison psychiatry) and whether evidence of alcohol or drug use was recorded for S136 detentions.

Secondary services for the purpose of the study referred to all services locally, both statutory and non-statutory, beyond those provided in primary care. Alcohol use was self-reported by the individual, objectively measured using blood alcohol levels or breathalyser, or by clinical signs of intoxication recorded by the police or approved mental health professional conducting the Mental Health Act assessment. Drug use was either self-reported by the individual or detected using urinary drug screening. Chi-squared analysis was used to examine the association between repeat S136 detention and diagnosis, and hospital admission and diagnosis. Mann-Whitney $U$ or Kruskal-Wallis tests (as appropriate) were used to investigate the relationship between diagnosis and total 
hospital admission days, and diagnosis and number of referrals to services. These non-parametric tests used as the continuous variables (total hospital admission days and number of referrals) were not normally distributed.

The data were collected and initially analysed using Microsoft Excel for Windows, and statistical tests were conducted using SPSS version 17 (SPSS, Chicago, Illinois, USA).

Using the NHS Health Services Authority online tool, it was determined that NHS Research Ethics Committee Approval was not required for this study.

\section{Results}

The demographics of the original cohort are shown in Table 1 , from the original paper. ${ }^{2}$ The most common primary diagnosis was F20F29 schizophrenia, schizotypal and delusional disorders $(n=52$, $21 \%)$, F60-F69 disorders of adult personality and behaviour ( $n=50$, $21 \%)$ and F10-F19 mental and behavioural disorders due to psychoactive substance use $(n=48,20 \%)$. Fifty-two per cent $(n=126)$ had either a primary or secondary diagnosis of psychoactive substance use (F10-F19).

Of the original S136 detentions, 59 (24\%) patients were detained under the Mental Health Act (52 under Section 2 and 5 under

Table 1 Demographic details of individuals detained

\begin{tabular}{|c|c|c|}
\hline & $n$ & $\%$ \\
\hline \multicolumn{3}{|l|}{ Age, years } \\
\hline $15-20$ & 29 & 11.8 \\
\hline $21-30$ & 63 & 25.7 \\
\hline $31-40$ & 50 & 20.4 \\
\hline $41-50$ & 64 & 26.1 \\
\hline $51-60$ & 29 & 11.8 \\
\hline$>60$ & 10 & 4.1 \\
\hline \multicolumn{3}{|l|}{ Gender } \\
\hline Male & 139 & 56.7 \\
\hline Female & 106 & 43.3 \\
\hline \multicolumn{3}{|l|}{ Ethnicity } \\
\hline White & 174 & 71 \\
\hline Black & 28 & 11.4 \\
\hline Asian & 18 & 7.4 \\
\hline Mixed & 11 & 4.5 \\
\hline Other & 8 & 3.1 \\
\hline Not stated & 6 & 2.5 \\
\hline \multicolumn{3}{|l|}{ Previous admission/section } \\
\hline Yes & 150 & 61.2 \\
\hline No & 89 & 36.3 \\
\hline Unknown & 6 & 2.5 \\
\hline \multicolumn{3}{|c|}{ Reason for Section 136 assessment } \\
\hline Threat of self-harm & 100 & 40.8 \\
\hline Bizarre behaviour & 50 & 20.4 \\
\hline Combination & 32 & 13.1 \\
\hline Actual self-harm & 29 & 11.8 \\
\hline Violence towards others & 28 & 11.4 \\
\hline Violence towards property & 4 & 1.6 \\
\hline Other & 2 & 0.8 \\
\hline a. Reproduced with permission of $Z$ & & \\
\hline
\end{tabular}

Section 3), 33 (14\%) were admitted informally, and 150 (62\%) were discharged to the community (36 (15\%) to a crisis resolution and home treatment team). No follow-up was considered necessary for $54(22 \%)$ individuals, 40 (16.5\%) were currently using secondary mental healthcare services, $26(11 \%)$ were referred to secondary care as a new patient, $16(6.6 \%)$ were out of area, $9(3.7 \%)$ were to be followed up in primary care and $5(2 \%)$ were discharged into police custody. Of the 40 individuals currently open to secondary care, 21 were to be followed up by the home treatment team, 10 by a community mental health team (CMHT), 6 by a drug and alcohol team (DAT), 2 jointly by a CMHT and a DAT, and 1 was a current in-patient. Of the 26 new referrals to secondary care, 15 were referred to the home treatment team, 8 to a CMHT, 2 to a DAT and 1 to both a CMHT and a DAT.

In the year following their original S136 detention, 41 individuals (17\%) were detained again by the police, and 201 (83\%) were not in South West London and St George's Mental Health NHS Trust. Most individuals $(n=39)$ were re-detained under S136 between one and four times; however, two individuals were detained under S136 over ten times. Current alcohol or drug use was recorded in 23 (56\%) of these repeat detentions. Over the follow-up period, 115 (48\%) individuals were admitted to hospital a total of 205 times. Current alcohol or drug use was recorded in 84 (41\%) of these 205 admission documents. Mostly this was a single admission ( $n=80)$; however, 35 individuals were admitted between 2 and 15 times. Ten patients were subject to a community treatment order (CTO). During the follow-up period, 62 (26\%) individuals were not referred to any secondary mental healthcare. Thirty (12\%) were referred once, 138 (57\%) between 2 and 10 times and 12 (5\%) were referred to services over 10 times. The median number of referrals per patient was two (including to liaison psychiatry services).

At the end of the year follow-up period, 106 (44\%) of the patients were under secondary care, 83 (34\%) were not under secondary care, $39(16 \%)$ were out of area, $9(4 \%)$ were in-patients, $3(1 \%)$ were deceased and $2(1 \%)$ were in prison. Of those using secondary care, 81 patients were under the care of a CMHT, 12 with a DAT, 10 with personality disorder services and 18 with other teams. Reasons for not using secondary care at 1 year were as follows: 39 did not have a mental disorder, 25 had disengaged or declined to use services, 6 were well and had been discharged, 6 had a primary diagnosis of alcohol use, 2 were using private psychiatric services and in 5 no reason was determinable. One patient had died by suicide, one died of liver failure and for one patient the cause was not recorded in the psychiatric notes. Figure 1 illustrates the outcomes at 1 year by the outcome of the original S136.

There was a significant association between diagnosis and repeat detention under $\operatorname{S136}\left(\chi^{2}(4, N=242)=29.32, P<0.001\right)$ (Table 2$)$. During the follow-up period, 18 (36\%) individuals with a primary diagnosis of personality disorder, $13(27 \%)$ with a psychoactive substance use disorder, 7 (13\%) with psychotic disorders, 2 (6\%) with affective disorders and one (2\%) other were re-detained under S136. The proportion of patients re-detained under S136 was significantly greater for those with a primary or secondary diagnosis of psychoactive substance use $(n=32,25 \%)$ compared with those without $(n=9,8 \%)\left(\chi^{2}(1, N=242)=13.35, P<0.001\right)$.

There was a significant association between diagnosis and hospital admission $\left(\chi^{2}(4, N=242)=44.66, P<0.001\right)$ and length of admission $\left(\chi^{2}(4, N=242)=15.77, P=0.003\right)$. Individuals with psychotic disorders were the most likely to be admitted ( $n=39,75 \%)$ and spend the most time as an in-patient (median total admission days=68.5) and individuals with psychoactive substance use the least likely $(n=8,17 \%)$ and they spent the least time as an in-patient (median total admission days=14). Having a primary or secondary diagnosis of psychoactive substance use was not associated with being admitted $\left(\chi^{2}(1, N=242)=0.731, P=0.392\right)$ or length of admission $(P=0.822)$. 


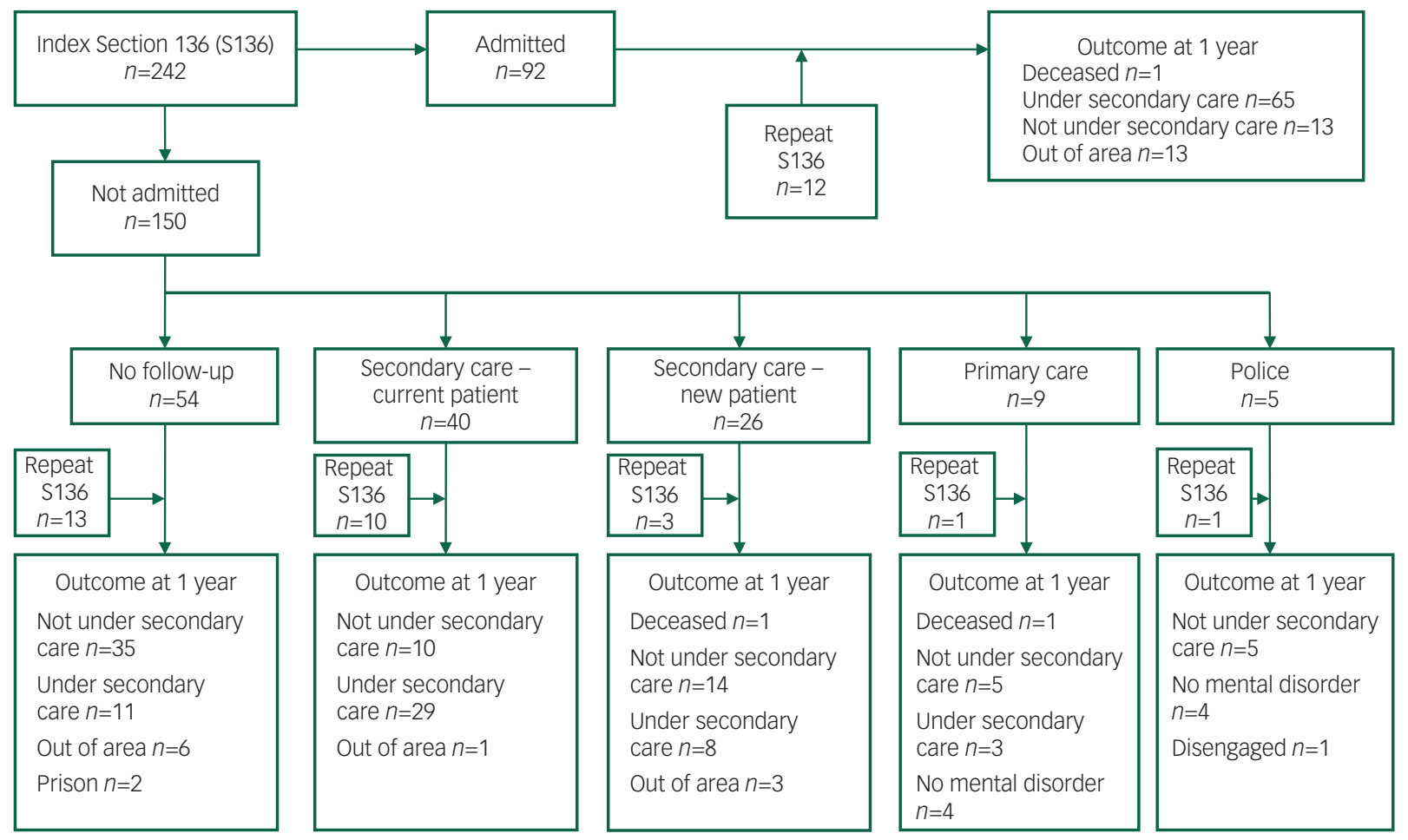

Fig. 1 Outcomes at 1 year by outcome of initial detention.

\begin{tabular}{|c|c|c|c|c|c|c|}
\hline & $\begin{array}{l}\text { Personality disorder } \\
\qquad N=50\end{array}$ & $\begin{array}{l}\text { Substance misuse } \\
\qquad N=48\end{array}$ & $\begin{array}{c}\text { Schizophrenia } \\
\qquad N=52\end{array}$ & $\begin{array}{l}\text { Affective disorder } \\
\qquad N=36\end{array}$ & $\begin{array}{l}\text { Other diagnosis } \\
\qquad N=56\end{array}$ & $P$ \\
\hline $\begin{array}{l}\text { Re-detained in follow-up period, } \\
n(\%)\end{array}$ & $18(36)$ & $13(27)$ & $7(13)$ & $2(6)$ & $1(2)$ & $\begin{array}{l}\chi^{2}=29.3 \\
P<0.001\end{array}$ \\
\hline Admitted in follow-up period, $n$ (\%) & $25(50)$ & $8(17)$ & $39(75)$ & $23(64)$ & $17(30)$ & $\begin{array}{l}\chi^{2}=44.7 \\
P<0.001\end{array}$ \\
\hline $\begin{array}{l}\text { Days admitted, mean, median } \\
\text { (range) }\end{array}$ & $27.6,0.5(0-173)$ & $4.5,0(0-73)$ & $82.5,30(0-366)$ & $23,17(0-139)$ & $1.5,0(0-272)$ & $\begin{array}{l}\chi^{2}=15.8 \\
P=0.003\end{array}$ \\
\hline Referrals made, median (range) & $4(0-27)$ & $2(0-13)$ & $3(0-9)$ & $2(0-9)$ & $0(0-7)$ & $\begin{array}{l}\chi^{2}=56.7 \\
P<0.001\end{array}$ \\
\hline
\end{tabular}

There was a significant association between diagnosis and the number of referrals made to secondary services $\left(\chi^{2}(4\right.$, $N=242)=56.74, P<0.001$ ). Those with a primary diagnosis of personality disorder were referred a median of four times, those with schizophrenia three times, those with affective and psychoactive substance use disorders twice and those with other diagnoses had a median of zero referrals.

\section{Discussion}

In 2014, the Department of Health and Home Office conducted a review of Sections 135 and 136. ${ }^{4}$ The Royal College of Psychiatrists responded that: 'Studies in the 1990s, mainly in London, suggested that the majority of those detained under S136 had schizophrenia, mania or drug-induced psychosis. However, current experience of members of the College suggests a greater proportion of those with personality disorder or chaotic behaviour complicated by substance misuse are now being detained under S136' (p. 67). The findings of this study support this observation: equal numbers of patients had primary diagnoses of psychosis, personality disorder and substance use, making up $60 \%$ of the sample. Over half had a primary or secondary diagnosis of psychoactive substance use. However, diagnosis seems to vary across time and location: a literature review found that the 'typical patient' had schizophrenia, ${ }^{5}$ in Manchester the most common diagnosis was mood disorder, ${ }^{6}$ and in Gloucestershire only 8 and $11 \%$ were diagnosed with personality disorder or substance use respectively. ${ }^{7}$

Over the follow-up period, the majority of patients (83\%) were only detained once, and most (52\%) were not admitted to hospital. However, there was a small group who presented multiple times to services. Patients with personality disorder were the most likely to be re-detained under S136, and those with a primary or secondary diagnosis of substance use were also more likely to be re-detained multiple times. A fifth of the patients were admitted to hospital twice or more, including one patient who was admitted 15 times in 1 year. This research did not analyse whether these two groups (high users of hospital and those frequently detained on S136) consist of the same patients, but given that those with psychosis were significantly more likely to be admitted than those with primary substance use disorders, they may be different. Nonetheless, in around half of the repeat detentions the individual had been using substances. Substance use should therefore be a target for interventions to reduce crisis contacts with health services and police. 
For half of the cohort, S136 was their route to either a hospital admission or a new referral to psychiatric services. The most common type of community follow-up was with the crisis and home treatment team. A year after the original detention, nearly half of patients were under secondary care. Although this suggests that these patients are correctly being identified by the police as having a mental health problem, patients can find contact with the police a punitive experience. ${ }^{8}$ It could be suggested that alternative pathways should be in place to avoid S136 detention as far as possible, especially in the $48 \%$ who are detained due to threat of self-harm. ${ }^{2}$ Despite the high numbers with personality disorder and substance use, at 1 year only 12 patients were with a DAT, and 10 were with personality services. Those with personality disorders were referred to the most number of individual services, indicating at best only short-term engagement with treatment.

This research supports the finding of Spence \& Mc Phillips, who conducted a small cohort study in Westminster, London. They also found that those with personality disorders were the most likely to be repeatedly detained, yet not admitted. A literature review noted that studies conducted in the 1980s had observed that those who presented to the police were 'frequently disorganized and unsupported, they had a high absconding and self-discharge rate, few were registered with a general practitioner and they were unlikely to attend follow-up. ${ }^{10}$ Although the members of the Royal College of Psychiatrists may think they have observed a change, perhaps not much has actually changed in the 25 years.

The strength of this study is that detailed information was available about detentions and service use, leading to very little missing data for those presenting within the area. However, the electronic notes system only records data for South West London and St George's Mental Health NHS Trust. This means we were unable to gather data for those patients, and information about services accessed, from out of area. Although the personality disorder services were run by the Trust during the study period, at least two drug and alcohol services were run by third-sector companies. This might have led to a potential underestimation of engagement with this treatment as the same electronic records were not shared. Some of the patients could have been accessing these services autonomously or via their general practitioner and we would not have captured this. This lack of information sharing not only presents a problem for research and service evaluation but may also present problems for the patient and their journey through services; an area possibly under-recognised and researched.

The local liaison psychiatry service uses the same electronic notes system, so we were able to record the number of referrals made by Accident and Emergency to the liaison team. This is a useful indication of how often these patients presented in crisis and used emergency services other than the police. As with other studies, the findings may not be applicable to other geographical areas.

This study shows that there are two potential routes to reducing the use of S136. A fifth of people detained under S136 can be said to have been unnecessarily detained, as they were discharged without follow-up after psychiatric assessment. Patients with personality disorders and substance use present repeatedly in crisis. Long-term care and treatments for these patients must be improved. The Department of Health and the Home Office ${ }^{4}$ have recommended that police should seek health advice (where feasible) before detention.
This could involve either increased joint working with the local crisis team or creating a street triage team. A study recently assessing two models of street triage (one with a mental health nurse working with front-line police, the other with nurses providing advice from the police control room) found that the model involving a nurse alongside front-line police showed significant reduction in S136 numbers. ${ }^{11}$

The local area has recently introduced a street triage team. This should be evaluated to determine whether it can reduce the use of S136 in this population, and whether it improves patient experience. In addition, more work should determine how and why patients already known to secondary care and detained due to self-harm come to the attention of police, and how these crises could be averted.

Jennifer L. Burgess, BA(Oxon), MBBS, Campus of Ageing and Vitality, Northumberland, Tyne and Wear NHS Foundation Trust, Newcastle upon Tyne, UK, and Institute of Neuroscience, Newcastle University, Newcastle upon Tyne, UK; SarahJane White, BSC, MSC, MRCPsych, MD, Population Health Research Institute, St George's University of London, London, UK; Aileen O'Brien, MBBS, BSC, MRCPsych, MD, Springfield Hospital, South West London and St George's Mental Health NHS Trust, London, UK, and St George's University of London, London, UK

Correspondence: Jennifer L. Burgess, Academic Psychiatry Department, Institute of Neuroscience, Newcastle University Wolfson Research Centre, Campus for Ageing and Vitality, Newcastle upon Tyne NE4 5PL, UK. E-mail: jennifer.burgess1@nhs.net

First received 7 Aug 2017, accepted 30 Sep 2017

\section{References}

1 Health and Social Care Information Centre. Inpatients Formally Detained in HoS pitals Under the Mental Health Act 1983 and Patients Subjective to Supervised Community Treatment: 2015/16, Annual Figures. NHS Digital, 2016 (http://www. content.digital.nhs.uk/catalogue/PUB22571/inp-det-m-h-a-1983-sup-com-eng15-16-rep.pdf)

2 Zisman S, O'Brien A. A retrospective cohort study describing six months of admissions under Section 136 of the Mental Health Act: the problem of alcohol misuse. Med Sci Law 2015; 55: 216-22.

3 World Health Organization. The ICD-10 Classification of Mental and Behavioural Disorders: Clinical Descriptions and Diagnostic Guidelines. WHO, 1992.

4 Department of Health and Home Office. Review of the Operations of Sections 135 and 136 of the Mental Health Act 1983: Review Report and Recommendations. TSO (The Stationery Office), 2014.

5 Borschmann RD, Gillard S, Turner K, Lovell K, Goodrich-Purnell N, Chambers M. Demographic and referral patterns of people detained under Section 136 of the Mental Health Act (1983) in a south London Mental Health Trust from 2005 to 2008. Med Sci Law 2010; 50: 15-18.

6 Sadiq K, Moghal A, Mahadun P. Section 136 assessments in Trafford Borough of Manchester. Clin Gov 2011; 16: 29-34.

7 Laidlaw J, Pugh D, Riley G, Hovey N. The use of Section 136 (Mental Health Act 1983) in Gloucestershire. Med Sci Law 2010; 50: 29-33.

8 Riley G, Freeman E, Laidlaw J, Pugh D. 'A frightening experience': detainees' and carers' experiences of being detained under Section 136 of the Mental Health Act. Med Sci Law 2011; 51: 164-9.

9 Spence SA, McPhillips MA. Personality disorder and police section 136 in West minster: a retrospective analysis of 65 assessments over six months. Med Sci Law 1995; 35: 48-52.

10 Borschmann RD, Gillard S, Turner K, Chambers M, O'Brien AO. Section 136 of the Mental Health Act: a new literature review. Med Sci Law 2010; 50: 34-9.

11 Jenkins $\mathrm{O}$, Dye $\mathrm{S}$, Obeng-Asare F, Nguyen $\mathrm{N}$, Wright $\mathrm{N}$. Police liaison and section 136: comparison of two different approaches. BJPsych Bulletin 2016; 41: 1-7. 